\title{
0854. Metabolomic changes by mass spectrometry in lung tissue from septic rats with mechanical ventilation-induced lung injury
}

\author{
Y Rojas ${ }^{1,2^{*}}$, S Naz ${ }^{3}$, N Nin ${ }^{4,5}$, A Garcia ${ }^{3}$, A Ferruelo ${ }^{1,2}$, L Martínez-Caro ${ }^{1,2}$, M de Paula ${ }^{1,6}$, C Barbas $^{3}$, JA Lorente ${ }^{1,2,7}$ \\ From ESICM LIVES 2014 \\ Barcelona, Spain. 27 September - 1 October 2014
}

\section{Objective}

To identify metabolomic changes in lung tissue associated with lung injury induced by mechanical ventilation (VILI) in animals with sepsis, using for the first time a global unbiased metabolomic fingerprinting approach.

\section{Methods}

Rats received cecal-ligation and puncture (CLP) or sham operation, and $24 \mathrm{~h}$ later underwent mechanical ventilation for $2.5 \mathrm{~h}$ with either $\mathrm{V}_{\mathrm{T}}=9 \mathrm{ml} / \mathrm{kg}$, positive end-expiratory pressure $(\mathrm{PEEP})=0 \mathrm{~cm} \mathrm{H}_{2} \mathrm{O}(\mathrm{n}=9$ and $\mathrm{n}=12$, without and with CLP, respectively); or $\mathrm{V}_{\mathrm{T}}=25 \mathrm{ml} / \mathrm{kg}, \mathrm{PEEP}=5 \mathrm{~cm}$ $\mathrm{H}_{2} \mathrm{O}$ ( $\mathrm{n}=13$ and $\mathrm{n}=12$, without and with CLP, respectively). Lung tissue samples were obtained and analyzed by nontargeted global fingerprinting approach for lung tissue analysis, applying multiple complementary analytical techniques, including liquid cromatography-mass spectrometry (MS), gas cromatography-MS, and capillary electrophoresis-MS. We followed the Principles of Laboratory Animal Care (2010/63/UE 22-09, RD 53/2013 BOE 1-02, ley 32/2007 BOE 7-11).

\section{Results}

Metabolomic changes characteristic of sepsis and VILI were identified. Lung tissue samples from septic rats with VILI were characterized by a specific metabolomic profile as compared to samples from septic rats without VILI. Metabolomic changes indicated increased oxidative stress, and changes in purine, energy, carnitine, aminoacid, urea cycle, vitamines, collagen, ceramide-sphingomyelin and phospholipid metabolism.

${ }^{1}$ Centro de Investigación Biomédica en Red de Enfermedades Respiratorias (CIBERES), Getafe, Spain

Full list of author information is available at the end of the article

\section{Conclusion}

A particular metabolomic profile can be identified in lung tissue from septic rats with lung injury induced by mechanical ventilation.

\section{Grant acknowledgment}

FIS 12/02898, FIS 11/02791, FIS 12/02451, European Network (7th FP) ITN 264864, CA11/00260.

\section{Authors' details}

${ }^{1}$ Centro de Investigación Biomédica en Red de Enfermedades Respiratorias (CIBERES), Getafe, Spain. ${ }^{2}$ Hospital Universitario de Getafe, Intensive Care Service, Getafe, Spain. ${ }^{3}$ Facultad de Farmacia, Universidad CEU San Pablo, Centro de Metabolómica y Bioanálisis (CEMBIO), Madrid, Spain. ${ }^{4}$ Hospital Español, Intensive Care Service, Montevideo, Uruguay. ${ }^{5}$ Hospital de Torrejon, Intensive Care Service, Madrid, Spain. ${ }^{6}$ Hospital Universitario de Getafe, Getafe, Spain. ${ }^{7}$ Universidad Europea de Madrid, Madrid, Spain.

Published: 26 September 2014

doi:10.1186/2197-425X-2-S1-P63

Cite this article as: Rojas et al:: 0854 . Metabolomic changes by mass spectrometry in lung tissue from septic rats with mechanical ventilationinduced lung injury. Intensive Care Medicine Experimental 2014 2(Suppl 1):P63.

Submit your manuscript to a SpringerOpen ${ }^{\odot}$ journal and benefit from:

- Convenient online submission

- Rigorous peer review

- Immediate publication on acceptance

- Open access: articles freely available online

- High visibility within the field

- Retaining the copyright to your article

Submit your next manuscript at $\boldsymbol{~ s p r i n g e r o p e n . c o m ~}$

\section{SpringerOpen $^{\odot}$}

C 2014 Rojas et al; licensee Springer. This is an Open Access article distributed under the terms of the Creative Commons Attribution License (http://creativecommons.org/licenses/by/2.0), which permits unrestricted use, distribution, and reproduction in any medium, provided the original work is properly cited. 